\section{PERIODIC PSYCHOSES*}

BY

\section{J. L. CRAMMER, $\dagger$ M.A., M.R.C.S., D.P.M. Department of Experimental Psychiatry, University of Birmingham}

The word "period" is most commonly used in medicine to describe the phenomenon of menstruation. "Periodic disease" is a name given to cases of regularly recurrent and intermittent episodes of fever, or oedema, or arthralgia, or gastric pain and vomiting, in which the attacks continue for many years without further development in otherwise healthy individuals (Reimann, 1951). By periodic psychoses I mean those mental illnesses which, upon study over a period of time, show a similar intermittent regularly recurring course, periods of depression or hypomania alternating with days or weeks of normal mood, attacks of excitement or agitation, episodes of stupor or confusional symptoms striking regularly by the calendar in seemingly mentally healthy or nearly healthy individuals. Such cases may be labelled manic-depressive illness, catatonic schizophrenia or schizo-affective state, sometimes even hysteria or psychopathy. Some occur in every mental hospital, though not always recognized as periodic, particularly when the outbursts or attacks come at longish intervals, perhaps every six weeks. However, they are easily discovered when looked for.

A periodic psychosis may be suspected when a patient is admitted and discharged twice or thrice in a year, since the regular intermittent nature of the illness means that there will be a fairly quick spontaneous recovery in hospital, and a relapse at home as soon as the next attack falls due. A chronic patient who, though usually well behaved, has times when he becomes aggressive or restless, or stays in bed all day, may prove on closer inquiry to be showing such behavioural change quite regularly and even predictably by the calendar. Nurses of experience are often much more aware of such patients, particularly in the back wards, than are the medical staff. In women, so-called menstrual psychoses must be suspect. Of course not all who suffer from periodic mood swings or other regularly intermittent psychiatric disability are in hospital, but exact information about these people is difficult to come by.

\section{Establishing the Periodicity}

The first step in any suspected case of periodic psychosis is to establish the period, the length of time between the onset of one attack, and the onset of the next. Where the medical notes give no guidance to the precise dates of past attacks the daily and nightly hospital ward reports, which are usually stored for a number of years, may be searched with profit, particularly if some sedative such as chloral or paraldehyde had to be given as an extra during the attack, since the use of a drug is fairly certain to be recorded. Or the report may indicate the patients kept in bed each day. If the illness is periodic, the dates of onset will fall approximately the same number of days apart, with occasional irregularities, rather like the menstrual cycle. Sometimes there is a gap twice or

* Read to the Section of Psychiatry at the Annual Meeting of the British Medical Association, Birmingham, 1958.

+ Now at the Department of Psychological Medicine, Royal Free Hospital, London. thrice the fundamental interval, or some other simple multiple, because one or more attacks have been missed without upsetting the fundamental rhythm of the disease.

Fig. 1 illustrates these points: the episodes, described as schizophrenic, are marked as black rectangles. During these episodes the patient stayed in bed, mumbled to himself, appeared to be hallucinated, showed peculiar mannerisms, was unable to reply intelligibly in speech or in writing to questions, and was restless and noisy at night. Between them he was up and about helping the nurses, in a competent and reliable manner, to run the ward, and was able to converse quite easily. He was in his early thirties when admitted
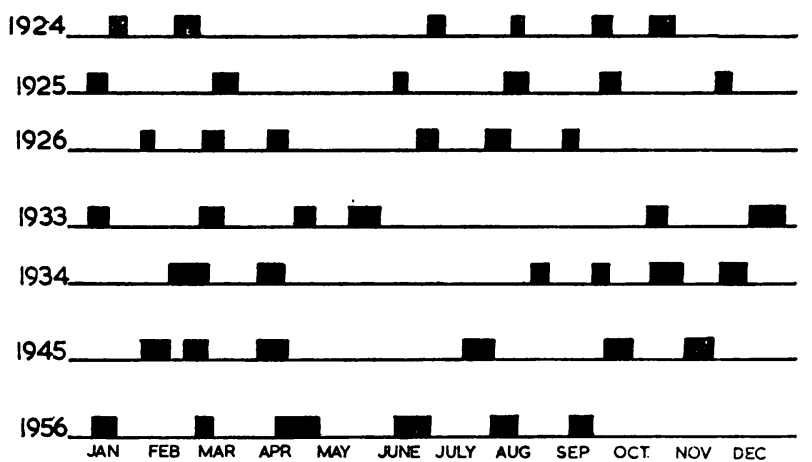

FIG. 1.-Episodes of schizophrenic catatonic disturbance in one patient over a number of years (the years not charted were similar). Some of the apparent gaps are likely to have been brief spontaneous remissions, others may have been a failure to record attacks.

to hospital, and in his old age became deaf and blind and bedridden. Nevertheless his attacks still continued with much the same frequency and duration as before. The earlier ones were charted from the data in the medical notes, the later ones from the ward records of sedation at night. One finds in such cases that restlessness at night and overactivity by day often precede by two or three days the full psychotic attack, and that there may also be a restless night or two when the attack has finished; this explains the slightly longer rectangles in 1956, based on the sedation record. The occasional gaps in the rows of black rectangles are due to the missed attacks.

There is a slight tendency for these gaps to be commoner in spring and summer, as Gjessing (1953) notes in his cases of periodic catatonic schizophrenia. I have found them in nearly all the cases I have studied, no matter what the clinical picture of the attack (Crammer, 1957). The most striking fact Fig. 1 shows is the stability of the disease: after 32 years it still ran on in the same old way, neither greatly worse nor greatly better. This is a characteristic of periodic psychoses: once the periodicity is established they do not develop further and produce fresh signs or symptoms. The most that happens is a quantitative change in the severity of some or all of the manifestations whose pattern makes the full attack.

I want to emphasize that such periodic cases occur in both sexes and in every hospital, albeit they are only a small minority of the in-patients. Such cases provide excellent opportunity for therapeutic test. Each patient continually runs through his cycle of behavioural and psychic change, and in any one individual the attacks are all alike, so that he can serve repeatedly as his own 
control. In studying the effect of a drug, for instance, one can discern which items of the clinical picture are altered and which untouched, and on stopping the drug see the full-blown clinical attack reappear, only to be altered when the drug is given again.

A periodic psychosis also offers the best opportunity in psychiatry for the discovery of a physical basis to mental symptoms and signs, that is to say, for finding some physical sign or pathological test helpful in diagnosis and in predicting the outcome of treatment, or some metabolic abnormality affecting the brain which is directly responsible for some or all of the symptoms.

\section{Physical Pathology}

The physical pathology of the periodic psychoses seems their most exciting aspect. The remainder of this paper is a brief view of this subject from general clinical study, from physical examination, and from metabolic experiment.

A survey of many cases gives at once a picture of diversity: diversity in the clinical pattern of the attack in different cases, diversity of rhythms, diversity in age of onset. While a four- or six-weekly rhythm seems not uncommon, shorter cycles are also found. MenningerLerchenthal (1950) found 52 cases in the literature with a two-day cycle, alternate days overactive and listless, for instance (see also Richter, 1938), and von Stockert (1957) has added another. These cases were variously diagnosed as schizophrenia or manic-depressive insanity, periodic catatonia, melancholia, and even neurasthenia. The age of the onset was in the twenties, the thirties, the forties, and even in the sixties and seventies. In some the condition was thought to be post-encephalitic, or syphilitic, or arteriosclerotic in origin; in others the result of a fright or a time of great anxiety; in yet others no preceding factor could be discovered.

Often the illness begins as an endogenous depression, or as schizophrenia, and the characteristic periodicity appears only after several months or a year or two. The clinical picture of the single attack in the fully established case is nearly always biphasic and shows first an episode of overactivity and then one of underactivity as its essential feature, whatever the diagnostic label. In the former the patient is restless and talkative and may be euphoric, but may show stereotyped thinking and behaviour. In the latter he is usually quiet or even stuporous, with loss of all feeling, has difficulty in concentrating and in volition, and may have fleeting paranoid ideas.

Different individuals show these various elements in very different proportions, so that one may apparently have recurrent attacks of mania only or of catatonic excitement only, another a series of attacks only of depression or stupor. However, close examination usually shows the other half of the biphasic pattern to be also present in minimal form. For example, in the case of a man of 75 with recurrent stupors (Crammer, 1957, Fig. 1) there was in fact a day or so's extremely mild talkative fidgetiness preceding each stupor by a few days. Fifty years earlier this man had suffered from recurrent excitements. With the passage of years these became less intense, and the brief quiet period after each attack enlarged and deepened, till finally he suffered from full stupor. Barnes (1909) reports the case of a man who took 16 years to accomplish this full changeover, which from published reports apparently occurs only in one direction, towards quieter attacks.
Within the biphasic outline of the acute attack the pattern is sometimes complex, involving shorter episodes of agitated or confusional behaviour for instance, and yet the whole pattern is repeated in sequence each time. Such atypical psychoses (neither truly schizophrenic nor manic-depressive) were separated off by Schröder (1926) as "degeneration psychoses" and by Kleist (1928) as "motility psychoses" and "confusional psychoses." It is important to understand that a periodic psychosis cannot be recognized as such at a single clinical interview, and only with difficulty after an acquaintance of a week or two. The simple and sure method is by the periodicity, which means a good history or at least a few months' recordings.

I have said that each attack is often a complex sequence of events, yet it is one which has an all-ornothing aspect to it; if it is missed it seems to be missed completely. This sequence is true for the physical phenomena, as well as for the mental. The patient may complain at some time of thirst, or vague abdominal discomfort, may have difficulty in passing urine, may develop a skin rash, and of course may show changes in facial appearance and eyelid motility, in sleep, in appetite, and in bowel habit. But these phenomena, if they occur, may only do so for a certain time within the whole sequence of the attack, and recur every attack only at the same relative time, peculiar to that case. If the attack is not carefully followed day by day to its end, these peculiarities may be missed. The same is true of such physical measures as pulse, blood pressure, temperature, and body weight. The total psychotic attack is elaborately orchestrated. Even where an attack appears clinically uncomplicated-for instance, a stuporous phase-drug experiments suggest cerebral changes while the patient's behaviour remains static and unchanging. It is well known that intravenous amylobarbitone sodium will awaken the stuporous catatonic. In the periodic case different doses are required on different days to produce the same effect. The depth of stupor varies on this measure, when clinically it appears level.

Descriptions of two cases we have been studying in Birmingham will illustrate these general statements.

\section{Case 1}

A man aged 49 has been in hospital for 10 years with a six-day cycle predominantly manic-depressive in type. Prior to this he seems to have been well, though probably of low intelligence to judge by his employment record. His illness began at the age of 38 with a manic attack which cleared. He was discharged home but a week later was readmitted again in mania, and has been in hospital since. It is not known exactly when his illness became cyclical, but records for the past five years show that it had not changed in that time, presenting always an uninterrupted succession of cycles each six to seven days long and consisting of about two days of a semi-stuporous state and four days of increasingly cheerful talkative overactivity.

In detail the cycles run like this. During the semi-stupor he sits all day staring into space, with a rather woebegone expression, doing nothing and saying nothing. To all questions he replies "yes," "no," or " dunno" in a low voice. He understands all instructions, but he is slow in all his movements. He eats a normal quantity of food, but very slowly. We have been able to awaken him temporarily from this state by intravenous injection of methyl phenidylate ("ritalin"), and he then passed briefly through a phase in which he expressed the view that his case was hopeless. Over a period of hours the semi-stupor begins 
to lighten. His expression becomes more alert, his responses quicker; he may pick up a newspaper and gaze at it; he begins to speak in a normal voice, single words, brief sentences, and, finally, may even venture an occasional spontaneous remark. He begins to smile.

Then there comes the moment when he stretches his arms and stands up, and his overactive phase has begun. For the first day or so he is able to walk a little, to sit down at times, to keep quiet on request, but he is soon walking about endlessly, with a mounting pressure of talk, buttonholing passers-by, laughing and joking. He stays awake all night, talking, singing snatches of songs. His talk becomes progressively loaded with obscenities and more erotic in content; he makes claims to being the greatest violinist in the world and the heir to wealth; but the range of his ideas is very narrow, and they are similarly and repetitively expressed in each cycle. As the hypomania reaches its height, his talk becomes so rapid that the words are slurred, some even omitted altogether. $\mathrm{He}$ talks endlessly and no longer requires any sort of audience. His expression ceases to be cheerful and smiling but becomes anxious and haggard. Then his talk changes more and more into mutter, ceases to have any pressure behind it; he walks up and down more slowly, smacking his lips; his attention becomes difficult to attract, and his answers to simple questions are erroneous. Finally he sits down, and the monosyllabic quiet phase has begun again.

Various bodily functions show linked cyclic change. Appetite remains almost constant throughout and fluid intake is steady-he refuses an extra drink even in his lip-smacking phase. But his bowels act only on the first two days of each overactive phase, when he sometimes complains of abdominal discomfort. His urine output shows a polyuria in the overactive phase and an oliguria during semi-stupor. Further, there is a period of difficulty in starting micturition, even with a full bladder, during the day of muttering that precedes the onset of
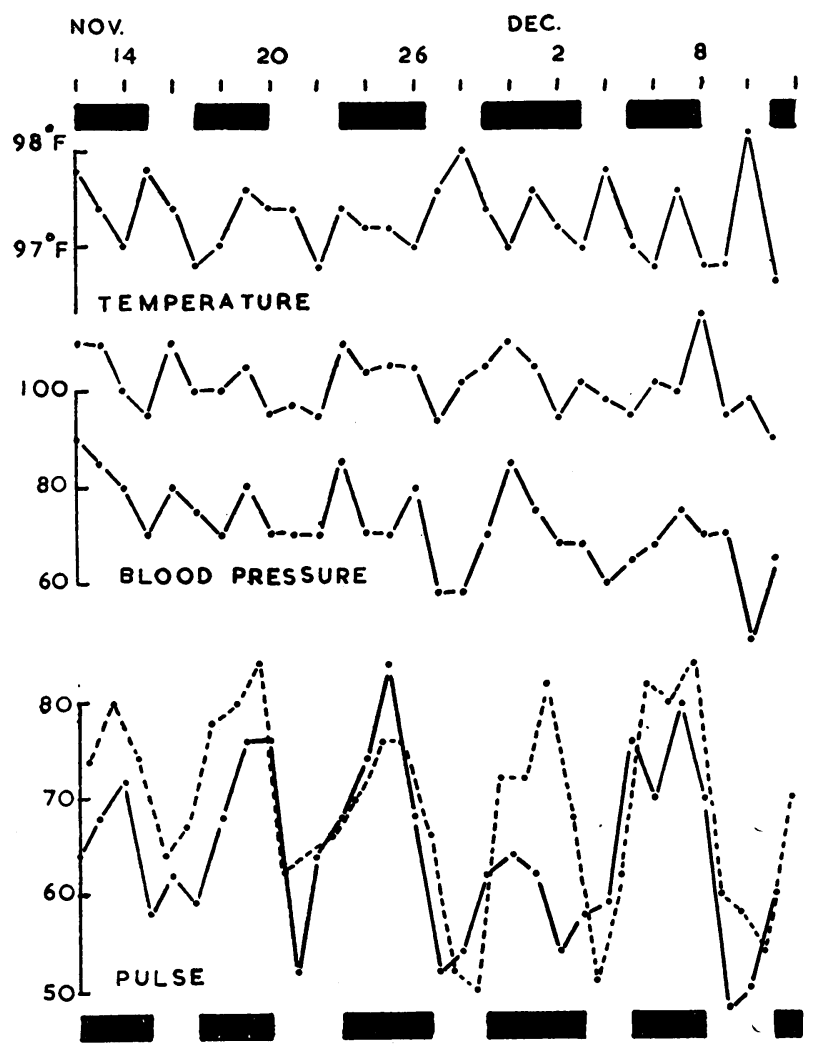

Fig. 2.-Case 1. Change in pulse with mental change: the pulse was taken in bed before rising in the morning (continuous line) and after retiring at night (dotted line). Active mental phases marked as black rectangles, the gaps between represent semi-stupor. semi-stupor, before the oliguric phase sets in, while one of the first signs that in a few hours he will liven up again is the sudden onset of the polyuria which precedes the overactivity. Two other physical signs are important. His pulse rate, counted resting in bed, shows large swings that bear no relation to his degree of overactivity (Fig. 2). His body weight measured daily shows a cyclic variation of about $5 \mathrm{lb}$. (2 kg.), rising during oliguria, falling during polyuria. By calculation and experiment we have been able to prove that his weight gain represents an increase in body water, and his weight loss is largely accounted for by excessive urine formation (Fig. 3).

\section{Case 2}

A man aged 48 first became ill in 1940 with an attack of depression, from which he recovered. It recurred in 1948 and lasted with variation in depth until in 1952 he had his first manic attack. Since then he has had a cyclic illness, approximately two we eks manic and two weeks depressed, with a brief
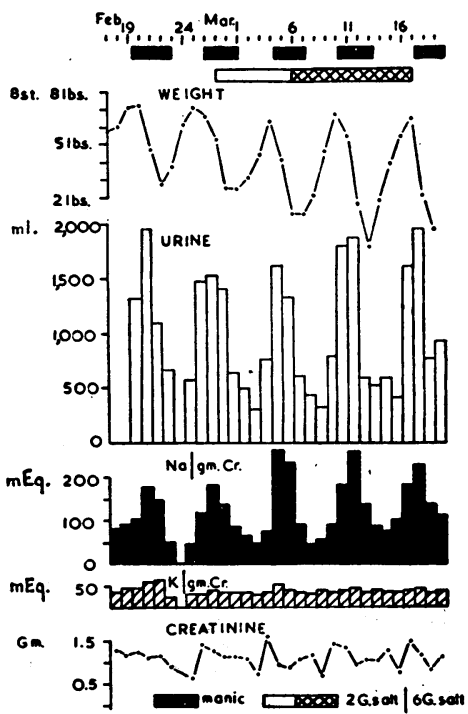

FIG. 3.- Case 1.-Fluctuation in body weight, with associated polyuria and oliguria and variable sodium excretion. Black rectangles represent active phases. Sodium and potassium expressed as per gramme of creatinine present in each urine specimen.

normal phase between each attack, so that the period from the onset of one manic phase to the onset of the next is about six weeks.

The manic phase begins suddenly. One day he is normal, the next he gets up in cheerful, smiling, talkative mood, which slowly warms up over a number of days with increasing overactivity and pressure of talk, ever-easier joking, laughter, and sociability, and some flight of ideas, and then slowly declines again. There are three or four approximately normal days, and then he becomes gradually but noticeably quieter, speaks only when spoken to, may have difficulty in understanding a question or in finding the answer to it, shows less spontaneous activity, and is inclined just to sit and look gloomy. This goes on for about four days. Then, quite sharply, a new phase is interposed. He stays in bed and refuses to get up, saying he cannot make up his mind about anything. He is restless in bed and picks at the sheets, he talks easily again and replies promptly and correctly; he smiles often, but his spirits are neither up nor down, but blank. After another four days this phase ends, and for about a day he appears suspicious and easily frightened, but denies any delusional ideas. He is quiet again, complains that everything costs him a great effort, and is slow. Gradually he improves, though he complains of difficulty in concentrating for a long time. He has about a week clear, and then the cycle resumes.

Bodily investigation shows no change in appetite, no striking change in bowel rhythm, though at the height of his depressive phase there is constipation. There is possibly a brief complaint of thirst during the body weight minimum in this phase. Pulse rate shows no cyclic change. Body weight remains steady during the overactive phase, and drops suddenly, about $5 \mathrm{lb}$. ( $2 \mathrm{~kg}$.) in all, just before the onset of the smiling restless depression, recovering itself in the tail of the depression. These weight changes are accompanied by polyuria and oliguria respectively, and must represent changes in body water content. 


\section{Comment}

In both cases when there is polyuria there is also a large loss of sodium and chloride in the urine, and in fact the magnitude of the body weight change seems to depend in part on the level of salt in the diet. Fluctuations in body weight have been noted as part of the attack in other periodic psychotics (Rowntree and Kay, 1952 ; Gjessing, 1953).

Scattered in the literature are reports of cases where mental change and episodic polyuria are associated. Klein and Nunn (1945) reported a case very similar to our six-day cycle man. Broser (1951) observed a man with approximately monthly disturbance of consciousness and brief coma, associated with oliguria, rise in body weight, and then a polyuria and weight fall, which he ascribed to a hypothalamic lesion. Biemond (1949) also described two cases of this sort. It seems not unlikely therefore that these sudden changes of body weight are the result of a hypothalamic disturbance in the course of mental illness, leading to under- or over-secretion of anti-diuretic hormone, and possibly also similar variation of an adrenocortical salt-retaining hormone.

Aldosterone is very difficult to measure at present, but 17-oxosteroids (17-ketosteroids), and the 17-hydroxycorticoids which mostly represent hydrocortisone secretion (Appleby et al., 1955), can be measured fairly satisfactorily. We found the 17-oxosteroids to be a very insensitive indicator of adrenal function; but the second group of steroids fluctuates cyclically in both our patients, being below their normal in the overactive state, and above it during the depressive phase (Fig. 4). This agrees with the
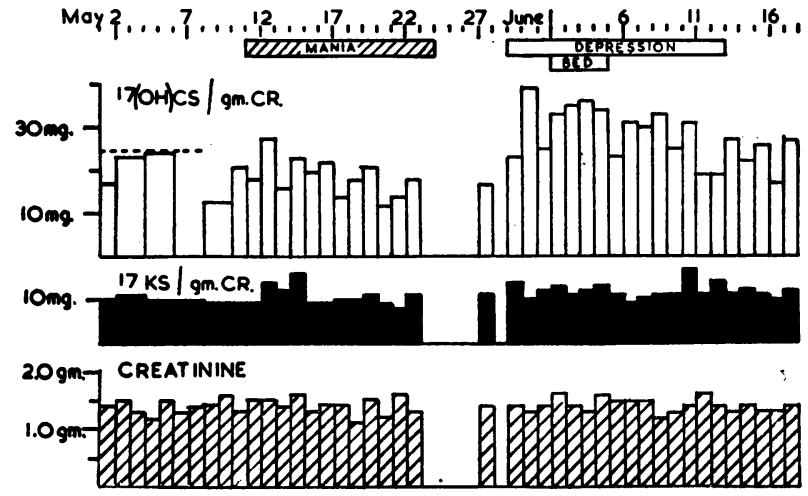

FIo. 4.-Total 17-hydroxycorticosteroid (17(OH)CS) and 17-ketosteroid excretion in successive 24-hour collections of urine through one manic-depressive attack. The dotted line on the left shows the average daily $17(\mathrm{OH}) \mathrm{CS}$ output during the previous month, when, exceptionally, the patient remained mentally normal.

finding of Rizzo et al. (1954) that adrenal cortical function was low in a manic woman and returned to normal as she recovered, although her glands were normally responsive to injected corticotrophin throughout her illness. The adrenal dances to the tune called by the hypothalamic-pituitary system. During the overactive phase the tune is soft, in the quiet phase it is loud.

\section{Discussion}

The painstaking work of Gjessing, in which he improved on the experiments of Pighini (1909) and Pighini and Statuti (1910), demonstrated conclusively that periodic catatonics show a cyclical retention and excretion of nitrogen in the form of urea. Gjessing's work unfortunately requires special facilities; the present paper concentrates on observations which can be made anywhere where there is conscientious nursing, a weighing machine, and a measuring cylinder; or at the most the facilities of an ordinary hospital laboratory. Further it concentrates on biochemical factors which may play a part in producing mental symptoms. It is well known that shifts in sodium and water produce mental symptoms. It is also known that adrenal cortical steroids may have such an effect. Since changes in both these chemical factors occur during the acute psychotic attack it is possible that they are responsible for part of its symptomatic pattern.

One can conceive of the mechanism of a periodic psychosis in three distinct parts: (1) the pacemaker, which sets the rhythm-only at regular intervals is a psychotic attack possible; (2) some factor which is required for the attack to be fired off; and (3) the symptomatic pattern of the psychotic attack, in which there is an ordered sequence of events, one physiological disturbance perhaps provoking the next-for example, a failure of secretion of enough antidiuretic hormone at one stage might result in cerebral dehydration at the next stage, and a fresh set of symptoms while this lasts. On this basis thyroid in a very large dose, known to be an effective treatment in periodic catatonia (Gjessing, 1935 ; Gornall et al., 1953), might act on the second factor, while chlorpromazine seems to act on the third part of the mechanism. We may hope that by pharmacological and biochemical analysis of this third part-the pattern of repeating psychotic attack-to test the hypotheses that electrolyte, water, and steroid changes are responsible for different parts of the symptom complex, and eventually perhaps to apply the knowledge gained to the study of non-periodic psychoses.

The single greatest need in this field of psychiatric research is that more work should be done, more cases studied by more people. I hope that what I have said may have done something to show that chronic patients, still unfortunately often a neglected group clinically, are worthy of careful study, and that this study can be carried on wherever people are willing to undertake it, without necessarily employing expensive apparatus or technical staff.

\section{Summary}

Patients suffering from periodic psychoses occur more commonly than is usually recognized. The illnesses are characterized by brief episodes of mental symptoms recurring regularly, often at monthly or six-weekly intervals, in both sexes. Their special importance is their suitability for research. A brief account is given of some leading clinical features. Some physical and biochemical findings in the two cases reported show how even with simple methods information useful for understanding the physical pathology of psychosis can be obtained.

I am grateful to Dr. J. J. O'Reilly and the medical and nursing staff of All Saints' Hospital, Birmingham, for the whole-hearted way they have aided our investigations.

\section{REFERENCES}

Appleby, J. I., Gibson, G., Norymberski, J. K., and Stubbs, R. D. (1955). Biochem. J., 60, 453

Barnes, F. M. (1909). Amer. J. Insan., 65, 591.

Biemond, A. (1949). Folia psychiat, neerl., 52, 343.

Broser, F. (1951). Arch. Psychiat., 187, 311.

Crammer, J. L. (1957). Lancet, 2, 259.

Gjessing, R. (1935). Arch. Psychiat., 104, 355. - (1953). Ibid., 191, 191. 
Gornall, A. G. Eglitis, B., Miller, A., Stokes, A. B., and Dewan, J. G. (1953). Amer. J. Psychiat., 109, 584 .

Klein, R., and Nunn, R. F. (1945). J. ment. Sci., 91, 79

Kleist, K. (1928). Schweiz. Arch. neurol. Psychiat., 23, 1.

Menninger-Lerchenthal, E. (1950). Wien. Z. Nervenheilk., 3, 261

Pighini, G. (1909). Arch. Neurol. (Lond.), 4, 220.

and Statuti, G. (1910). Amer. J. Insan., 67, 299.

Reimann, H. A. (1951). Medicine (Baltimore), 30, 219

Richter, C. P. (1938). Arch. Neurol. Psychiat. (Chicago), 39, 587.

Rizzo, N. D., Fox, H. M., Laidlaw, J. C., and Thorn, G. W. (1954). Ann. intern. Med., 41, 798 .

Rowntree, D. W., and Kay, W. W. (1952). J. ment. Sci., 98, 100 Schröder, P. (1926). Z. ges. Neurol. Psychiat., 105, 539

Von Stockert, F. G. (1957). Nervenarzt, 28, 445.

\section{TRIFLUOPERAZINE DIHYDROCHLORIDE ("STELAZINE") IN PARANOID SCHIZOPHRENIA}

\author{
BY
}

RODERICK MACDONALD, M.B., Ch.B., D.P.M. Physician-Superintendent, Holloway Sanatorium, Virginia Water

AND

T. P. SHIELDS WATTS, M.B., B.S. Registrar, Holloway Sanatorium, Virginia Water

Experience of phenothiazine derivatives in this hospital had prior to November, 1957, been mainly confined to chlorpromazine, which had been given in doses of up to $850 \mathrm{mg}$. daily together with electric convulsion therapy (E.C.T.). On the whole it can be said that some relief in symptomatology was obtained in patients with chronic schizophrenia, rather better success in patients suffering from involutional paranoid illness, but the results in cases of acute recent schizophrenia were minimal to moderate and did not obviate the necessity for insulin coma treatment (I.C.T.). It would be fair to criticize our experience with chlorpromazine in that perhaps we were too cautious, as better results have been reported with intensive chlorpromazine plus E.C.T. (Ollendorff, 1958). Nevertheless it appeared that a drug of similar action, more powerful, less toxic, and simpler in its administration, might give better or equally good results without the necessity for E.C.T. or, in acute cases, without the need for I.C.T.

Trifluoperazine dihydrochloride ("stelazine") was reputed to be such a drug. It is derived from prochlorperazine by the substitution of a trifluormethyl group for the chlorine atom in the 2 position of the nucleus. According to the manufacturers it is reputed to be four times as powerful as prochlorperazine and between nine and ten times as powerful as chlorpromazine in blocking conditioned responses in animals.

In November, 1957, a trial of trifluoperazine was begun and its effect tested on a series of patients suffering from acute and chronic paranoid schizophrenia. The patients were treated under the conditions normally applying in this hospital, and no other treatment was given during the experiment except occasional night sedation, and all proceeded with the normal programme of rehabilitation.

The results of treatment in the first 50 patients so treated in the first nine months of the experiment are here recorded. They were divided into two groups.

\section{Methods and Material}

Group A.-There were 30 male patients suffering from chronic paranoid schizophrenia who had been in this hospital for a minimum period of two years. All had previously been treated by standard methods, such as I.C.T. and E.C.T., and had been given a prolonged trial with chlorpromazine and, in many cases, other tranquillizers available at the time. None of these treatments had materially altered the clinical picture. The duration of the illness varied from two to fourteen years.

Group B.-There were 20 patients admitted during the first attack of schizophrenia of less than six months' duration who had not had any previous treatment. They were admitted with a recommendation for I.C.T. by other consultants, and when initially examined in this hospital were thought to be cases that would normally have proceeded to I.C.T.

Treatment and Dosage.-Treatment was begun by suspending all other drugs. It was continued by administering $5 \mathrm{mg}$. of trifluoperazine daily for the first three days, thereafter increasing by $5 \mathrm{mg}$. daily at threeday intervals until gross side-effects appeared or a satisfactory therapeutic effect had been obtained. When the symptoms had been controlled by trifluoperazine the dosage was reduced to a maintenance level, on which patients were completely free from side-effects and at which the therapeutic effect seemed maximal. The maximum dose used was $45 \mathrm{mg}$. daily and the average maintenance dose 20-25 mg. daily. Those discharged have been maintained on dosages varying from 5 to 20 mg. daily.

\section{Results}

Assessment of the effect of trifluoperazine was made on the patient's capacity towards adjustment to normal social surroundings. These results are shown in the accompanying Table in terms of the degree of deterioration before and after treatment.

\begin{tabular}{|c|c|c|c|c|c|c|}
\hline Group & & & (a) & (b) & (c) & (d) \\
\hline A \{ & $\begin{array}{l}\text { Before tro } \\
\text { After }\end{array}$ & " & $\begin{array}{r}26 \\
9\end{array}$ & $\begin{array}{c}4 \\
12^{*}\end{array}$ & $\overline{4}$ & $\overline{5}$ \\
\hline B \{ & $\begin{array}{l}\text { Beforo } \\
\text { After }\end{array}$ & ", & $\frac{14}{-}$ & $\begin{array}{l}6 \\
6 \dagger\end{array}$ & 二 & $\overline{14}$ \\
\hline
\end{tabular}

* Two of these are fit for discharge but waiting placement

+ Three of these six made good initial improvement with trifluoperazine but proceeded to I.C.T. on the advice of other psychiatrists before trifluoperazine was given a full trial.

(a) Incapable of social living and requiring constant observation. (b) Able to adjust to social living in the hospital community (insluding parole, short leaves of absence, etc.). (c) Discharged from hospital and able to adjust socially but incapable of earning a living. (d) Discharged from hospital with return to full employment.

Follow-up.-All patients discharged have continued with a maintenance dose. No patients in Group B have reported a relapse. Three in Group A showed a return of symptoms when they had difficulty in obtaining the drug, but they quickly responded when the drug was recommenced. All have been out of hospital for from five to nine months.

Side-effects.-The only side-effects noted were, in order of frequency: drowsiness, Parkinsonism, increased motor restlessness, and increased salivation. No significant alterations were observed in blood pressure, white-cell counts, liver-function tests, or urinary analysis. Drowsiness and salivation occurred on dosages from $10 \mathrm{mg}$. daily, increased motor restlessness from $15 \mathrm{mg}$., and Parkinsonism of $20 \mathrm{mg}$. and above. The most troublesome side-effects, from the patients' point of view, were drowsiness and motor restlessness. The side-effects 